\title{
Induction of LFA-1-Dependent Neutrophil Rolling on ICAM-1 by Engagement of E-Selectin
}

\author{
BETSY C. CHESNUTT, ${ }^{*}$ DAVID F. SMITH, ${ }^{\dagger}$ NIKOLAI A. RAFFLER, ${ }^{\ddagger}$ MICHAEL L. SMITH, \\ E. J. WHITE, ${ }^{*}$ AND KLAUS LEY ${ }^{* \dagger, \dagger}$ \\ *Department of Biomedical Engineering, ${ }^{\dagger}$ Departments of Molecular Physiology and Biological \\ Physics, and ${ }^{\ddagger}$ Robert M. Berne Cardiovascular Research Center, University of Virginia, \\ Charlottesville, Virginia, USA
}

\begin{abstract}
Objective: To study rolling of mouse neutrophils on E-selectin and ICAM-1 in an ex vivo flow chamber system.

Methods: The authors developed a small autoperfused flow chamber $(20 \times 200-\mu \mathrm{m}$ cross section) that allows direct visualization of cells with and without fluorescent labeling and does not require recirculation of blood.

Results: Neutrophils rolled on E-selectin alone, but were unable to interact with immobilized ICAM-1. When ICAM-1 was co-immobilized with E-selectin, the number of cells that rolled was doubled, but no significant firm adhesion was observed. This phenomenon was specific for E-selectin, and no enhancement of rolling was observed when P-selectin was immobilized with ICAM-1. The increased neutrophil rolling seen on E-selectin and ICAM-1 substrates required $\beta_{2}$ integrins. Treating mice with antibodies to the $\beta_{2}$ integrins LFA-1 and Mac-1 showed that LFA-1 was primarily responsible for mediating rolling on ICAM-1 in this model. Increased rolling on E-selectin and ICAM-1 was significantly reduced following administration of a specific p38 mitogen-activated protein kinase (MAPK) inhibitor.

Conclusion: The data show that neutrophil rolling on E-selectin leads to partial activation of LFA-1, enabling LFA-1-dependent rolling on ICAM-1. This mechanism is likely to amplify and accelerate neutrophil recruitment in inflammation.

Microcirculation (2006) 13, 99-109. doi:10.1080/10739680500466376
\end{abstract}

KEY WORDS: E-selectin, ICAM-1, LFA-1, neutrophil, rolling

To access inflamed tissue, circulating leukocytes must tether to the endothelium, roll, become firmly adherent, and transmigrate through the vessel wall. Rolling is mediated primarily by selectins $(4,22,31)$ and $\alpha_{4}$ integrins $(1,3) . \beta_{2}$ integrins are known to contribute

We thank Tom Graf for the gracious donation of the lysM-GFP mice. We also thank Becky Paska for technical help with the flow chambers. This work was supported by NIH EB 02185.

The current affiliation of Betsy C. Chesnutt is the Joint Program in Biomedical-Engineering, University of Memphis and University of Tennessee-Memphis, Memphis, TN 38152, USA.

The current affiliation of Michael L. Smith is BiologischOrientierte Materialwissenschaften, ETH-Hönggerberg, 8092 Zürich, Switzerland.

Address correspondence to Klaus Ley, MD, University of Virginia, Robert M. Berne Cardiovascular Research Center, P.O. Box 801394, Charlottesville,VA22908-1394,USA.E-mail:klausley@ virginia.edu

Received 4 August 2005; accepted 21 September 2005. to slow rolling in combination with selectins and can support rolling upon partial activation $(9,10,24,41)$. As leukocytes roll, chemokines and other signaling pathways activate integrins, leading to firm adhesion $(5,8,16,46)$. It is not known whether the partial integrin activation necessary for rolling on ICAM- 1 is induced by incomplete chemokine-dependent activation or by selectin engagement.

Rolling on selectins allows leukocytes to come in contact with other factors, such as chemokines, that can induce activation and lead to firm adhesion $(24,33)$. There is also evidence that selectin ligation can directly mediate integrin activation. Cross-linking L-selectin on the surface of human neutrophils enhanced the ability of chemotactic stimuli to activate $\beta_{2}$ integrins $(16,50)$. Furthermore, when isolated human neutrophils were perfused over a monolayer of E-selectin and ICAM-1 expressing L-cells, $60 \%$ of the interacting cells became firmly adherent, and adhesion was inhibited by blocking the $p 38$ 
mitogen-activated protein kinases (MAPK) (46). In these studies isolated human neutrophils were used. which were partially activated by the isolation procedures. Ficoll- or Percoll-based neutrophil isolation leads to an increase in Mac-1 expression and decrease in L-selectin expression $(13,15,27)$. Previous studies have shown that neutrophils perfused over selectin substrates in whole blood roll at much higher wall shear stresses than isolated neutrophils $(31,45,49)$.

Several in vivo studies suggest that E-selectin may have a role in the conversion of rolling to firm arrest. Milstone et al. demonstrated that E-selectin-deficient mice treated with TNF- $\alpha$ had normal numbers of rolling leukocytes, but impaired firm arrest (35). When formyl-methionyl-leucyl-phenalanine (fMLP) was microinjected next to a venule in the cremaster muscle of an E-selectin-deficient mouse, firm adhesion was reduced by more than $80 \%$ as compared with wild-type mice (33).

It has recently been demonstrated that integrins undergo conformational changes upon activation. In its resting state, LFA-1 is in a bent conformation and has low affinity for ligand. In the fully activated state, the integrin is in an extended conformation with an open headpiece, and it has high affinity for ligand and supports firm adhesion. Between these two lies an intermediate activation state in which the integrin is in the extended conformation, but the headpiece is closed $(2,44)$. Salas et al. used allosteric inhibitors to stabilize LFA-1 in various conformations and demonstrated that this intermediate activation state can mediate leukocyte rolling on ICAM-1, while the fully open conformation preferentially mediates firm adhesion $(40,41)$. Isolated LFA-1 I-domains have been shown to support rolling on high density ICAM-1 and ICAM-3 (26). These studies indicate that LFA-1 can support leukocyte rolling and firm adhesion, that rolling is mediated through I-domain interactions with ICAM-1, and that the interplay between rolling and firm adhesion may be controlled by the conformation and activation state of the integrin headpiece. However, the physiological mechanisms that can induce this intermediate activation state and allow $\beta_{2}$ integrins to support rolling remain to be identified.

In vitro flow chamber assays have been used for many years to study the function of leukocyte adhesion molecules. These systems allow individual molecules to be examined in an environment that mimics the hemodynamic state in blood vessels, without many of the complicating factors present in vivo $(7,21,29$
31,46). Flow chambers have been used to identify and characterize many aspects of the leukocyte adhesion cascade, including the role of selectins in capturing neutrophils from free flow and integrins in subsequent firm adhesion (31), the ability of $\alpha_{4}$ integrins to mediate mononuclear cell rolling $(1,3)$, and the importance of chemokines for leukocyte firm adhesion $(5,14,17)$. Recently, autoperfused flow chambers directly coupled to the carotid artery and jugular vein of a mouse were described $(18,49)$. Such flow chambers that are coupled to animals ex vivo have an advantage over traditional parallel plate flow chambers because they do not require leukocyte isolation.

Although these autoperfused flow chambers overcome many limitations of the traditional parallel plate flow chamber, they still have shortcomings. It is difficult to control wall shear stress and generate high wall shear stresses. Blood is recirculated following perfusion through the flow chamber. Because of the thickness of the chamber $(200 \mu \mathrm{m})$ and the presence of red blood cells, leukocytes interacting with the flow chamber surface can be identified only if they are fluorescently labeled (49). To overcome these limitations, we reduced the cross-sectional area of our flow chamber to $20 \times 200 \mu \mathrm{m}$. This allows direct observation of unlabeled leukocytes, allows the wall shear stress to be controlled, and reduces blood volume lost to nanoliters, obviating the need to recirculate blood.

The present study was undertaken to investigate how E-selectin engagement influences neutrophil interaction with ICAM-1. We show that E-selectin induces rolling on ICAM-1, thus providing a natural amplification mechanism for inflammation.

\section{MATERIALS AND METHODS}

\section{Recombinant Proteins and Antibodies}

Recombinant murine P-selectin/Fc, E-selectin/Fc, and ICAM-1/Fc were obtained from R\&D Systems (Minneapolis, MN), and keratinocyte-derived chemokine (KC) from Peprotech (Rocky Hill, NJ). The blocking monoclonal antibody ( $\mathrm{mAb}$ ) 9A9 (rat $\operatorname{IgG}_{1}, 30 \mu \mathrm{g} /$ mouse) against murine E-selectin was provided by B. Wolitzky (MitoKor, San Diego, CA). The Mac-1 mAb M1/70 (rat $\operatorname{IgG}_{2 b}, 30 \mu \mathrm{g} /$ mouse) was purchased from PharMingen (San Diego, CA). The LFA-1 mAb TIB-217 (rat IgG ${ }_{2 a \kappa}, 30 \mu \mathrm{g} /$ mouse) was purified at the Lymphocyte Culture Center at the University of Virginia from hybridoma supernatant (ATCG, Rockville, MD). The p38 MAPK inhibitor 
SB203580 (100 $\mu \mathrm{g} /$ mouse, administered for $1 \mathrm{~h}$ ) was purchased from Sigma-Aldrich (St. Louis, MO) (51).

\section{Animals}

All animal experiments were performed under a protocol approved by the animal care and use committee of the University of Virginia. Animals were housed in a barrier facility under specific pathogenfree (SPF) conditions, and all experiments were performed on 8- to 20-week-old mice that appeared healthy.

Transgenic mice with an enhanced green fluorescent protein (eGFP) gene inserted into the lysosome M (lys) locus were provided by T. Graf (Albert Einstein College of Medicine, New York, $\mathrm{NY}$ ). LysM-GFP ${ }^{+}$mice have been shown to express fluorescence on cells of the myelomonocytic lineage (12). LysM-GFP mice used in this study were backcrossed into the $\mathrm{C} 57 \mathrm{Bl} / 6$ line for at least 10 generations.

Gene-targeted mice lacking CD18 (CD18-/- ) (42) were crossed with lysM-GFP mice to generate CD18-/-/lysM-GFP mice that do not express any $\beta_{2}$ integrins. Murine genomic DNA was analyzed using PCR for the wild-type and knockout CD18 alleles and to detect the lysM-GFP allele. The wild-type allele was amplified using a forward primer from exon 2 of murine CD18 (5'-CTG GAC TGT TCT TCG TGG GAT C-3') and a reverse primer from exon $3\left(5^{\prime}-\right.$ GTA CTT GGT GCA TTC CTG GGA C-3'), yielding a 400-bp product. The CD18 knockout allele was detected using the same forward primer and a reverse primer from the inserted neomycin resistance gene (5'-AGA TCA TCG TGA TCG ACA AGA CG-3'). PGR conditions for the detection of the CD18 wild-type and knockout alleles were $94^{\circ} \mathrm{C}$ for 4 min, 35 cycles of $94^{\circ} \mathrm{C}$ for $60 \mathrm{~s}, 57^{\circ} \mathrm{C}$ for $60 \mathrm{~s}$, and $72^{\circ} \mathrm{C}$ for $60 \mathrm{~s}$, followed by $72^{\circ} \mathrm{C}$ for $7 \mathrm{~min}$. The lysM-GFP transgenic mouse PCR assay used forward and reverse primers that amplify within the enhanced green fluorescent protein (eGFP) gene. The forward primer $\left(5^{\prime}\right.$-TAA ACG GCC ACA AGT TCA GCG-3') and the reverse primer (5'-TAC TCG AGC TTG TGG CGC AGG ATG TT-3') yielded a 390-bp product. PCR conditions for the detection of lysM-GFP were $94^{\circ} \mathrm{C}$ for $4 \mathrm{~min}, 30$ cycles of $94^{\circ} \mathrm{C}$ for $30 \mathrm{~s}, 59^{\circ} \mathrm{C}$ for $30 \mathrm{~s}$, and $72^{\circ} \mathrm{C}$ for $30 \mathrm{~s}$, followed by $72^{\circ} \mathrm{C}$ for $7 \mathrm{~min}$.

\section{Flow Chamber Assembly}

Flow chambers were constructed from rectangular glass capillaries with a cross section of $20 \times 200 \mu \mathrm{m}$
(VitroCom, Mountain Lakes, NJ). Each chamber was cut to $30 \mathrm{~mm}$ using a glass cutting stone (Hampton Research, Aliso Viejo, CA). The chamber was placed between two plastic microscope coverslips (SigmaAldrich, St. Louis, MO) and attached to a glass microscope slide (Sigma-Aldrich, St. Louis, MO) using fingernail polish (Del Laboratories, Uniondale, NY). The chamber was inserted into a piece of heparinized polyethylene (PE) 50 tubing (ID $0.58 \mathrm{~mm}$, OD $0.965 \mathrm{~mm}$, Becton Dickinson, Sparks, MD) 5 $\mathrm{cm}$ in length. The joint was sealed with two-part epoxy gel resin (Loctite, Manco, Avon, OH). Adhesion molecules and chemokines dissolved in PBS $(3 \mu \mathrm{g} / \mathrm{mL}$ E-selectin, $1.5 \mu \mathrm{g} / \mathrm{mL}$ ICAM- $1,30 \mu \mathrm{g} / \mathrm{mL}$ P-selectin, $5 \mu \mathrm{g} / \mathrm{mL} \mathrm{KC)}$ were allowed to adsorb onto the glass surface for $2 \mathrm{~h}$ at room temperature, and then the chambers were incubated with $10 \%$ casein in PBS (Pierce Chemicals, Dallas, TX) for $1 \mathrm{~h}$. The free end of the chamber was then inserted into a piece of saline-filled PE 50 tubing $(150 \mathrm{~cm}$ length), and the joint was glued with two-part epoxy gel resin. The water-filled tubing was used to control the pressure at the downstream end of the flow chamber.

\section{Ex Vivo Microscopy}

Mice were anesthetized with an intraperitoneal (IP) injection of ketamine hydrochloride $(125 \mathrm{mg} / \mathrm{kg}$, Sanofi Winthrop Pharmaceuticals, New York, NY), xylazine $(12.5 \mathrm{mg} / \mathrm{kg}$, TranquiVed, Phoenix Scientific, St. Joseph, MO), and atropine sulfate $(0.025 \mathrm{mg} / \mathrm{kg}$, Fujisawa USA, Deerfield, IL) and placed on a heating pad maintained at $37^{\circ} \mathrm{C}$. The trachea of each mouse was intubated using PE 90 tubing (ID $0.86 \mathrm{~mm}$, OD $1.27 \mathrm{~mm}$ ), and the left jugular vein and carotid artery were cannulated using PE 10 tubing (ID $0.28 \mathrm{~mm}$, OD $0.61 \mathrm{~mm}$ ). The cannulas were flushed with $10 \mathrm{U} / \mathrm{mL}$ heparin in saline before each flow chamber was connected. A $40-\mu \mathrm{L}$ aliquot of blood was drawn from the carotid artery, and differential leukocyte counts were measured using an automated blood cell counter (Hemavet 850FS, CDC Technologies, Oxford, CT).

Microscopy was conducted on a Zeiss Axioskop intravital microscope (Carl Zeiss, Thornwood, $\mathrm{NY}$ ) with a saline immersion objective (SW 20/0.5). Flow chambers were illuminated using stroboscopic flash epi-illumination (Strobex 236, Chadwick Helmuth, Mountain View, CA) and/or halogen transillumination. Images were recorded with a CCD camera (model SIT66, DAGE-MTI, 
Michigan City, IN) connected to a Panasonic S-VHS recorder.

\section{Calculation of Wall Shear Stress}

The average wall shear stress $\left(\tau_{\mathrm{w}}\right)$ was calculated from the pressure drop as

$$
\tau_{\mathrm{w}}=\frac{\Delta P w h}{2 L(w+h)}
$$

where $\Delta P$ is the measured pressure drop across the chamber (in $\left.\mathrm{cm} \mathrm{H}_{2} \mathrm{O}\right), w$ is the width of the chamber $(200 \mu \mathrm{m}), h$ is the height of the chamber $(20 \mu \mathrm{m})$, $L$ is the length of the chamber $(30 \mathrm{~mm})$, and $\tau_{\mathrm{w}}$ is the average wall shear stress across the flow chamber cross section (49). Because the hydraulic diameter of the flow chamber $\left(D_{\mathrm{h}}=0.036 \mathrm{~mm}\right)$ is smaller than the inner diameter of the PE $10(\mathrm{ID}=0.28 \mathrm{~mm})$ or PE $50(\mathrm{ID}=0.58 \mathrm{~mm})$ tubing and resistance to flow is inversely proportional to the radius to the fourth power, almost all of the pressure drop occurs across the flow chamber and not in the tubing. Therefore, the pressure drop is equal to the difference between the mouse's arterial blood pressure and the pressure created downstream of the flow chamber by a water column.

This was verified by calculating the pressure drop in the tubing and across the flow chamber for a fluid with a known viscosity, water. If water was perfused at a flow rate of $2 \mu \mathrm{L} / \mathrm{min}$, the total pressure lost in the tubing would be $0.39 \mathrm{~cm} \mathrm{H}_{2} \mathrm{O}$, while the pressure drop across the flow chamber would be $21 \mathrm{~cm}$ $\mathrm{H}_{2} \mathrm{O}$, indicating that approximately $98 \%$ of the total pressure is lost across the flow chamber.

The average wall shear stress calculation uses the continuum approximation and assumes that the entrance length, $L_{\mathrm{e}}$, is much less than $L$ and the flow is quasi-steady (49). The continuum approximation is valid for blood flowing in glass capillary tubes with diameters as small as $20 \mu \mathrm{m}$ (34), and the hydraulic diameter of the rectangular flow chambers used here is $36 \mu \mathrm{m}$. Because flow from the carotid artery is pulsatile, the Womersley number, $W_{0}$, was used to determine if flow was quasi-steady. Flow is considered quasi-steady if $W_{0}$ is less than one. The Womersley number in the micro flow chamber was 0.8 , indicating that flow is quasi-steady.

The pressure drop across the flow chamber was controlled by an adjustable water column connected to the downstream end of the flow chamber. For the experiments reported in this study, the water column was adjusted to maintain an average wall shear stress of $5-7 \mathrm{dyn} / \mathrm{cm}^{2}$, values similar to the shear stress seen in vivo in postcapillary venules (48).

\section{Data Analysis}

Video recordings were digitized into a Macintosh computer using a MicroMotion DC30 video compression card (Pinnacle Systems, Mountain View, CA) and Adobe Premiere software (Adobe Systems, San Jose, CA). Velocities were measured with the public domain program NIH Image (http://rsb.info.nih.gov/ nih-image/) using custom written macros similar to those previously described (36). Ten rolling leukocytes were measured per field of view (FOV) at each time point. For statistical analysis, data were evaluated using Student's $t$ test. Statistical significance was considered for $p$ values at $p<.05$.

\section{RESULTS}

\section{Autoperfused Micro Flow Chamber}

An autoperfused flow chamber 100 times smaller in cross section than previous models was developed that allows direct visualization of cells without the use of fluorescence (Figure 1). Although transillumination is possible, epifluorescence and lysM-GFP mice are used in most experiments to positively identify neutrophils. This flow chamber also allows the wall shear stress to be controlled over a wide range and eliminates the need to recirculate blood. If blood was allowed to perfuse each flow chamber for $15 \mathrm{~min}$ at the maximum flow rate, the total blood lost per chamber would be only $54 \mu \mathrm{L}$, or about $2 \%$ of the blood volume of the mouse. Therefore, blood recirculation becomes unnecessary.

\section{Neutrophil Rolling on E-Selectin and ICAM-1}

To determine the effect of ICAM-1 on neutrophil rolling on selectins, E-selectin and ICAM-1 were immobilized alone or together on glass micro flow chambers. LysM-GFP mice were used to examine the interactions of neutrophils with the substrate. Consistent with previous reports (39), $89 \pm 3 \%$ of the cells rolling on E-selectin and $89 \pm 2 \%$ of those rolling on E-selectin/ICAM-1 were brightly fluorescent neutrophils.

When E-selectin was immobilized alone, after 5 min of perfusion $18 \pm 3$ cells rolled per field of view (FOV), and no firm adhesion was observed 


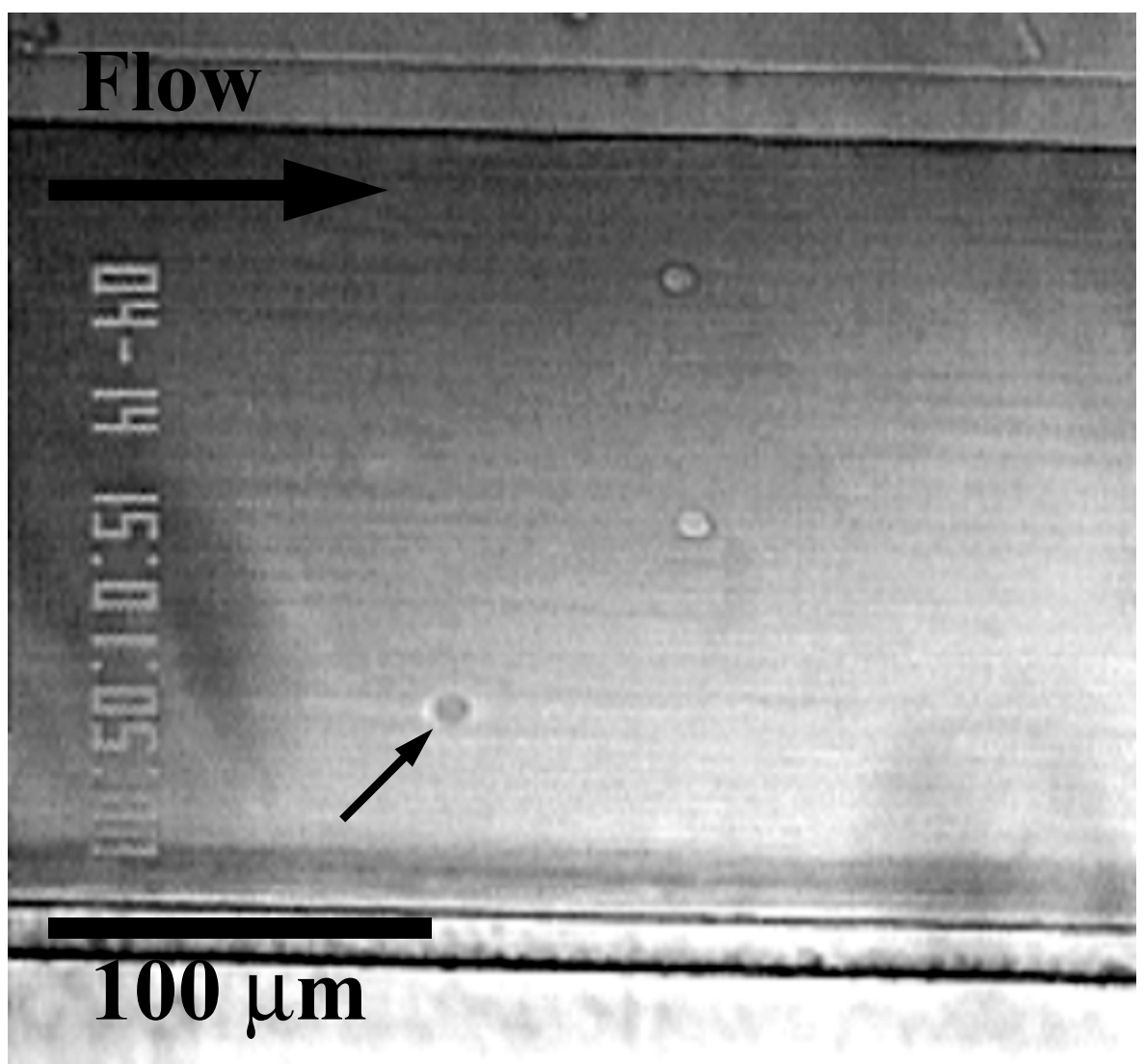

Figure 1. Analysis of the auto-perfused micro flow chamber. Cells rolling in the auto-perfused flow chambers can be visualized with or without fluorescent labeling. The picture shows nonfluorescent leukocytes (indicated by arrows) rolling on P-selectin, visualized by halogen transillumination. Scale bar $=100 \mu \mathrm{m}$.

(Figure 2). When ICAM-1 was co-immobilized with E-selectin, the number of rolling cells increased to $39 \pm 6$ cells/FOV, with no significant firm adhesion. ICAM-1 alone was unable to support any rolling or adhesion (Figure 2 and data not shown). Rolling was ablated on both E-selectin and co-immobilized Eselectin/ICAM- 1 by pretreating the mice with 9A9, an E-selectin blocking antibody (38). Although site densities of E-selectin and ICAM-1 could not be determined due to the small size of the flow chamber, the E-selectin site density cannot be higher when ICAM1 and E-selectin were co-immobilized than when Eselectin was immobilized alone. Theoretical maximum site densities were calculated by assuming that all of the protein in the flow chamber immobilized (Table 1). Enhanced ICAM-1-dependent rolling was specific for E-selectin, because the number of rolling cells was similar when P-selectin was immobilized alone $(17 \pm 5$ cells/FOV) or P-selectin and ICAM-1 were co-immobilized $(17 \pm 6$ cells/FOV, Figure 3$)$.

\section{Influence of ICAM-1 on Rolling Velocities}

To examine the effect of ICAM-1 on the rolling velocity of cells rolling on $\mathrm{E}$ - or P-selectin, the velocities of leukocytes rolling on E-selectin, Eselectin/ICAM-1, P-selectin, and P-selectin/ICAM-1 substrates were measured (Figure 4). Leukocytes rolled slowly on E-selectin $(1.25 \pm 0.07 \mu \mathrm{m} / \mathrm{s})$, consistent with previous reports indicating that E-selectin mediates slow leukocyte rolling in vivo (22). The addition of ICAM-1 did not change the velocity of rolling leukocytes $(1.28 \pm 0.09 \mu \mathrm{m} / \mathrm{s})$ (Figure 4A). When ICAM-1 was co-immobilized with P-selectin, however, a significant decrease in rolling velocity was observed from $5.1 \pm 0.6$ to $1.9 \pm 0.2 \mu \mathrm{m} / \mathrm{s}$ (Figure 4B). This is consistent with an earlier study demonstrating that the presence of immobilized ICAM-1 could decrease the rolling velocity of human neutrophils rolling on P-selectin without inducing an increase in the total number 


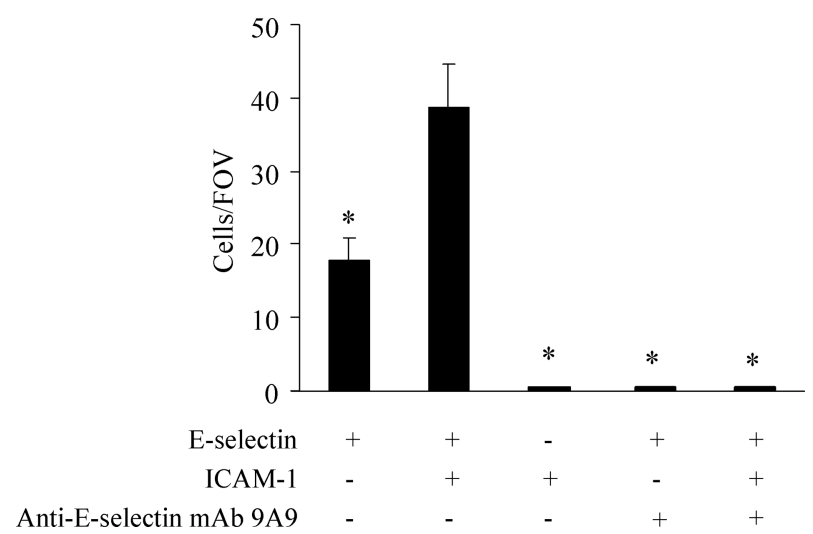

Figure 2. Neutrophil rolling on E-selectin and ICAM-1. E-selectin $(3 \mu \mathrm{g} / \mathrm{mL})$, ICAM-1 $(1.5 \mu \mathrm{g} / \mathrm{mL})$, or both Eselectin and ICAM-1 were co-immobilized in micro flow chambers. All measurements were made 5 min after perfusion of blood began. The number of cells that rolled on E-selectin/ICAM-1 was doubled compared to E-selectin alone. Mice pretreated with 9A9 (anti-E-selectin antibody) showed significantly reduced rolling counts on indicated substrates. $n=3-10$ mice for each substrate used. $* p<.05$ when compared to untreated cells rolling on $\mathrm{E}$ selectin/ICAM-1.

of rolling cells or any significant firm adhesion (8).

\section{$\beta_{2}$ Integrins in Neutrophil Rolling on E-Selectin and ICAM-1}

To investigate the role of $\beta_{2}$ integrins in neutrophil accumulation and rolling on E-selectin and ICAM-1, mice deficient in CD18 were crossed with lysMGFP mice to produce CD18-/- mice (42) with fluorescent neutrophils (CD18-/-/lysM-GFP mice). CD18-/-/lysM-GFP mice have elevated neutrophil counts $(42)$. When blood from CD18-/-/ysM-GFP mice was perfused through flow chambers with coimmobilized E-selectin and ICAM-1, the number of rolling cells per FOV was not significantly dif-

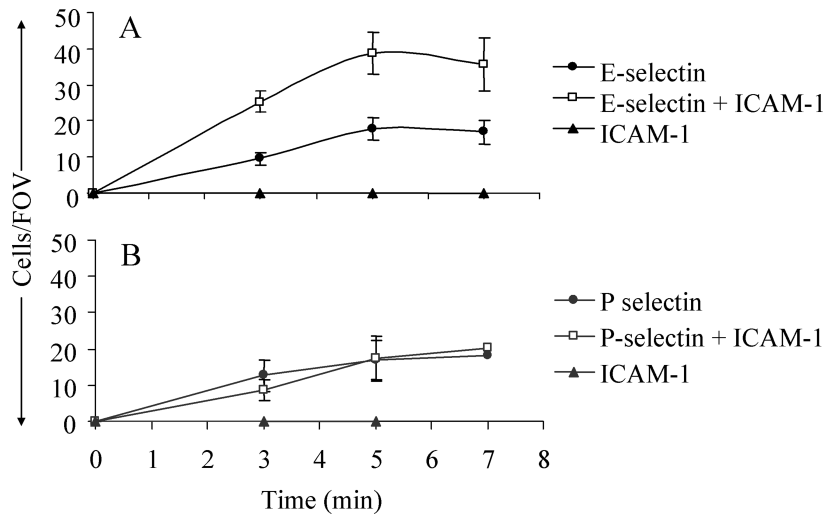

Figure 3. Neutrophil accumulation on E-selectin or Pselectin with and without ICAM-1. (A) Accumulation of rolling neutrophils on co-immobilized E-selectin (3 $\mu \mathrm{g} / \mathrm{mL})$ and ICAM-1 $(1.5 \mu \mathrm{g} / \mathrm{mL})$ was increased twofold over the accumulation seen when E-selectin was immobilized alone. No cells rolled when ICAM-1 was immobilized alone $(n=10$ mice). (B) There was no difference in the number of cells that rolled on P-selectin $(30 \mu \mathrm{g} / \mathrm{mL})$ or co-immobilized P-selectin $(30 \mu \mathrm{g} / \mathrm{mL})$ and ICAM-1 $(15 \mu \mathrm{g} / \mathrm{mL})(n=3$ mice$)$.

ferent from the number of rolling cells when Eselectin was immobilized alone (Figure 5A). This suggests that the increased accumulation of neutrophils on E-selectin and ICAM-1 is dependent on $\beta_{2}$ integrins.

To further examine the contribution of specific $\beta_{2}$ integrins to neutrophil recruitment, antibodies to LFA-1 and Mac-1 were administered intravenously to lysM-GFP mice, and the recruitment of neutrophils to co-immobilized E-selectin and ICAM-1 was examined (Figure 5B). A specific Mac-1 mAb, M1/70, decreased the number of rolling neutrophils slightly but not significantly. Administration of TIB 217, a mAb specific to LFA-1, significantly reduced the number of rolling neutrophils to $12.8 \pm 2$ cells/FOV, and when mice were treated with both TIB217 and M1/70, the number of rolling neutrophils was

Table 1. Theoretical site densities

\begin{tabular}{|c|c|c|c|c|c|c|}
\hline & $\begin{array}{c}\text { Coating } \\
\text { conc. } \\
(\mu \mathrm{g} / \mathrm{mL})\end{array}$ & $\begin{array}{c}\text { Molecular } \\
\text { weight } \\
(\text { g/mol })\end{array}$ & $\begin{array}{l}\text { Flow chamber } \\
\text { volume } \\
(\mu \mathrm{L})\end{array}$ & $\begin{array}{l}\text { Surface } \\
\text { area } \\
\left(\mu \mathbf{m}^{2}\right)\end{array}$ & $\begin{array}{c}\text { Total } \\
\text { protein } \\
\text { molecules }\end{array}$ & $\begin{array}{c}\text { Theoretical maximum } \\
\text { site density } \\
\left(\text { sites } / \mu \mathrm{m}^{2}\right)\end{array}$ \\
\hline E-selectin & 3 & 85,800 & 0.12 & $1.32 \times 10^{7}$ & $2.53 \times 10^{9}$ & 191 \\
\hline ICAM-1 & 1.5 & 76,800 & 0.12 & $1.32 \times 10^{7}$ & $1.41 \times 10^{9}$ & 106 \\
\hline P-selectin & 30 & 99,100 & 0.12 & $1.32 \times 10^{7}$ & $21.9 \times 10^{9}$ & 1657 \\
\hline
\end{tabular}

Note. Theoretical maximum site densities of immobilized E-selectin/Fc, P-selectin/Fc, and ICAM-1/Fc were calculated by assuming that all of the protein in the flow chamber immobilizes on the surface. 


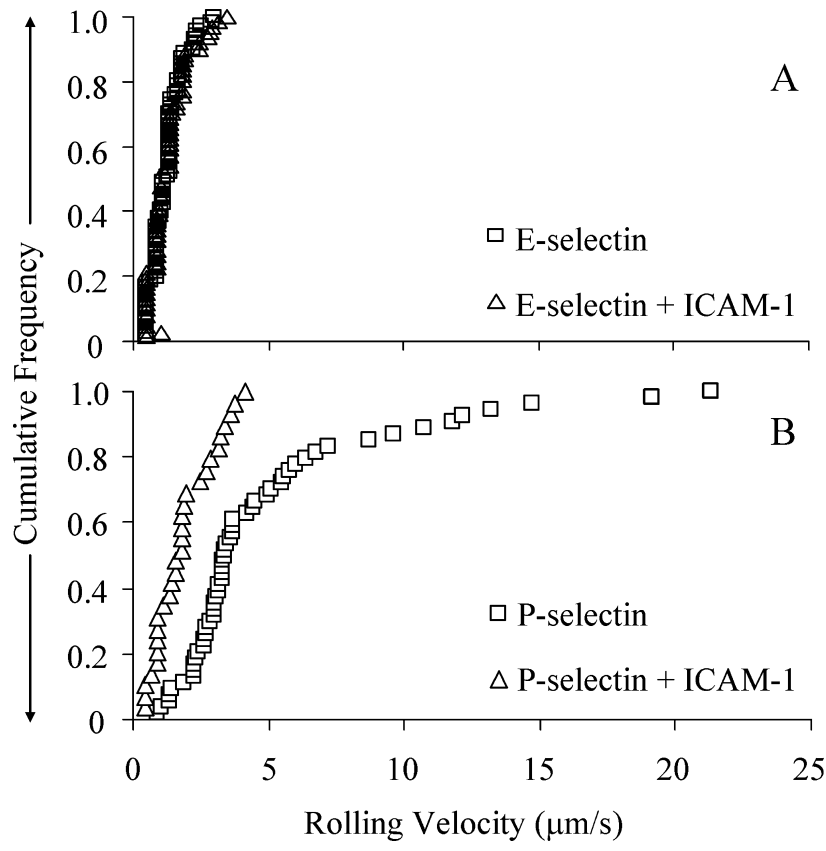

Figure 4. Cumulative histograms of rolling velocities of cells rolling on E-selectin, P-selectin, and ICAM-1. The velocities of at least 50 cells rolling on E-selectin (A) or Pselectin (B) with or without ICAM-1 were measured. Cells rolled more slowly on P-selectin/ICAM-1 (1.9 $\pm 0.2 \mu \mathrm{m} / \mathrm{s})$ than on P-selectin alone $(5.1 \pm 0.6 \mu \mathrm{m} / \mathrm{s})$. Adding ICAM1 reduced the rolling velocity on $\mathrm{P}$-selectin but not on $\mathrm{E}$ selectin.

$9.5 \pm 4$ cells/FOV. This is similar to the number of cells that rolled when ICAM-1 was blocked $(7.0 \pm$ 1 cells/FOV). Taken together, these results indicate that LFA-1 is the most important $\beta_{2}$ integrin for neutrophil recruitment and rolling on E-selectin/ICAM-1 substrates.

\section{p38 MAPK Signaling in Neutrophil Rolling on E-Selectin and ICAM-1}

Cross-linking of L-selectin or tethering to E-selectin on transfected cells was shown to induce activation of $\beta_{2}$ integrins and firm adhesion of neutrophils, and this adhesion was dependent on p38 MAPK signaling $(20,46,50)$. To determine if the increased neutrophil rolling observed on E-selectin/ICAM-1 substrates was dependent on MAPK signaling, the p38 MAPK inhibitor SB203580 was administered to lysM-GFP mice and accumulation of neutrophils on E-selectin- and ICAM-1-coated flow chambers was observed.

Treatment with SB203580 induced mild neutrophilia. The number of cells rolling on E-selectin,

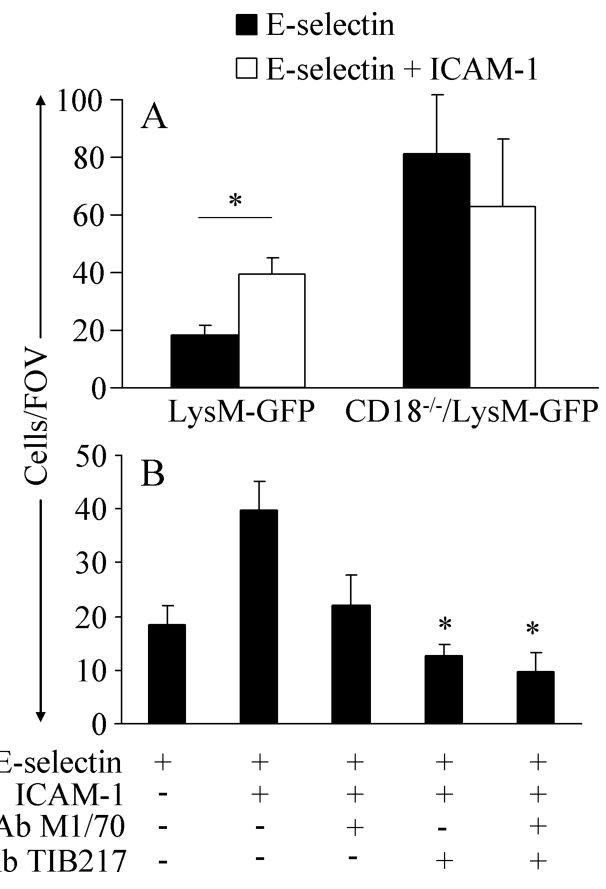

Figure 5. Participation of $\beta_{2}$ integrins in neutrophil rolling on E-selectin/ICAM-1 substrates. (A) Rolling and eliminated enhancement by ICAM-1 in CD18 ${ }^{-/-} /$lysMGFP mice. Increased numbers of neutrophils in CD18-/-/lysM-GFP mice have been reported earlier

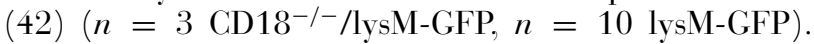
(B) TIB-217, an LFA-1 blocking antibody, significantly reduced the number of cells that rolled on E-selectin and ICAM-1, while M1/70, an antibody specific for Mac-1, did not significantly reduce the number of cells that rolled. $(n=8$ mice for E-selectin, $n=10$ mice for E-selectin/ICAM-1, $n=6$ mice for E-selectin/ICAM$1+\mathrm{TIB} 217+\mathrm{M} 1 / 70, n=3$ for others). ${ }^{*} p<.05$.

normalized to systemic neutrophil count, was $12 \pm$ 2 cells/FOV, compared to $25 \pm 5$ cells/FOV when Eselectin and ICAM-1 were co-immobilized (Figure 6). Following administration of SB203580, the normalized number of rolling cells was significantly reduced to $9 \pm 3$ cells/FOV. These results indicate that the increased accumulation of rolling neutrophils on coimmobilized E-selectin and ICAM-1 requires p38 MAPK signaling.

\section{Firm Adhesion of Neutrophils Induced by Immobilized Chemokines}

Immobilized chemokines have been shown to induce neutrophil firm arrest in several in vitro flow chamber assays (8,32). To determine if immobilized KC could induce firm adhesion of rolling neutrophils in the autoperfused micro flow chamber, 


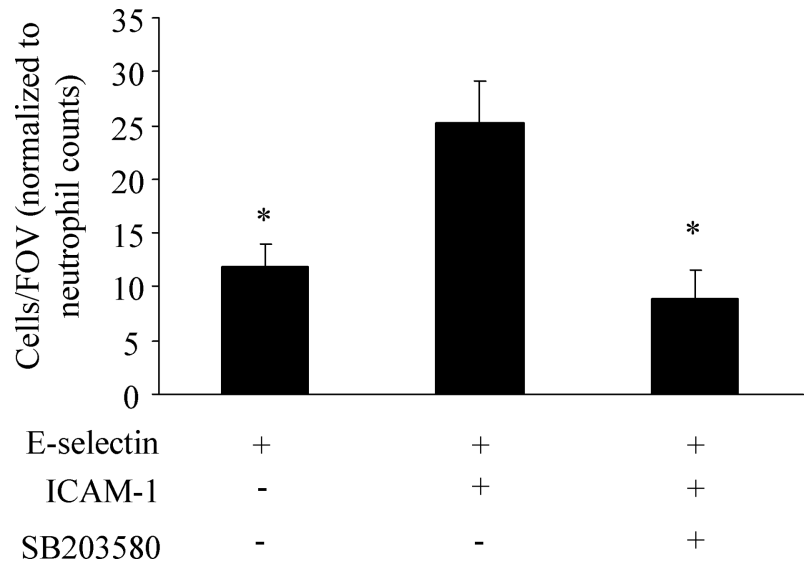

Figure 6. Inhibition of p38 MAPK signaling. Following treatment with SB203580, a specific inhibitor of p38 MAPK signaling, the number of rolling cells normalized by the systemic neutrophil count rolling on E-selectin and ICAM-1 decreased significantly after inhibition of p38 MAPK signaling. ${ }^{*} p<.05$.

KC was co-immobilized with E-selectin and ICAM-1 (Figure 7A). KC caused a significant increase in the number and percentage of adherent cells when compared with an E-selectin/ICAM-1 substrate
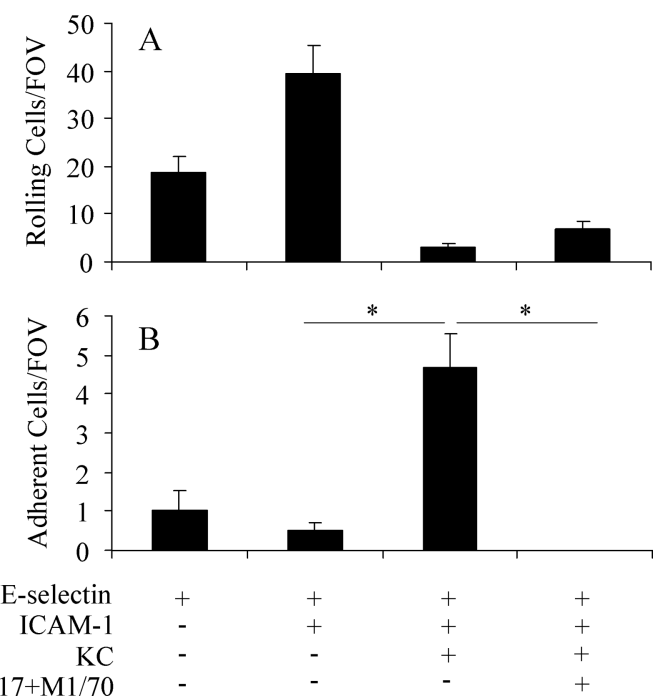

mAbs TIB217+M1/70

Figure 7. Firm adhesion induced by immobilized chemokines. A chemokine known to induce neutrophil firm arrest in vitro, $\mathrm{KC}$, was co-immobilized with $\mathrm{E}$-selectin and ICAM-1. (A) In the presence of KC, the total number of rolling was decreased. (B) There was a significant increase in the number of firmly adherent neutrophils when KC was co-immobilized with E-selectin and ICAM- 1 . When the $\beta_{2}$ integrins LFA-1 and Mac-1 were blocked by the antibodies TIB217 and M1/70, respectively, firm adhesion was completely blocked.
(Figure 7B). To confirm that firm adhesion was dependent on activation of $\beta_{2}$ integrins, mice were treated with the mAbs TIB217 and M1/70 against LFA-1 and Mac-1, respectively. After administration of antibodies, no leukocytes were able to make the transition from rolling to firm arrest (Figure 7B). When P-selectin was co-immobilized with ICAM-1 we detected a similar increase in adherent cells in the presence of co-immobilized KC (data not shown).

\section{DISCUSSION}

This study introduces an improved autoperfused micro flow chamber that allows direct visualization of murine leukocytes with and without fluorescence. Because the autoperfused flow chamber presented in this study is about 100 times smaller in cross-sectional area than previous flow chambers, rolling cells can be visualized using transillumination. Furthermore, the interactions of fluorescent and nonfluorescent cells can be observed using a combination of brightfield and fluorescence microscopy. Blood does not have to be recirculated because blood loss per flow chamber is minimal. This completely eliminates the possibility that leukocytes activated by passage through the flow chamber could enter the systemic circulation. The autoperfused flow chamber presented in this study allows the wall shear stress to be directly controlled. In other autoperfused flow chambers, the wall shear stress could be measured, but not directly controlled (49). An adjustable water column on the downstream end of the flow chamber controls the pressure drop across the micro flow chamber, allowing wall shear stresses from 0 to $29 \mathrm{dyn} / \mathrm{cm}^{2}$ to be generated.

The autoperfused flow chamber was used to investigate synergism between E-selectin and ICAM-1 in neutrophil recruitment and rolling. We utilized the flow chamber approach to investigate the effects of individual molecules on adhesion and rolling without the complications in vivo and to dissect out the specific effects of E-selectin. When E-selectin and ICAM-1 were co-immobilized on the surface of the flow chamber, the number of rolling cells was doubled compared to E-selectin alone. In high endothelial venules of Peyer's patches the site density of ICAM- 1 was estimated to be 13,800 sites $/ \mu \mathrm{m}^{2}{ }^{\circ}(6)$. In our experiments we found that an approximately 140-fold lower density was sufficient for notable adhesion. P-selectin may be expressed at around 2050 sites $/ \mu \mathrm{m}^{2}$ on cultured endothelial cells (19). No absolute numbers for the in vivo site densities of 
E- or P-selectin are available, although relative measurements have been performed by several groups $(11,19,25)$. Site densities used in our study produced reproducible and stable rolling and adhesion. The rolling velocities in the flow chamber are within the range that has been found in vivo, where the majority of the rolling cells in TNF- $\alpha$ treated cremaster venules have velocities below $5 \mu \mathrm{m} / \mathrm{s}$ and about $25 \%$ are below $2 \mu \mathrm{m} / \mathrm{s}(23,28)$.

This study shows, for the first time, that E-selectin and ICAM-1 synergistically recruit neutrophils without inducing firm adhesion. The synergism observed was shown to be specific for E-selectin and ICAM-1. It was further determined that the number of neutrophils that rolled on E-selectin/ICAM-1 substrates was greatly reduced by inhibition of the p38 MAPK signaling pathway. The p38 MAPK pathway has been shown to be important in many inflammatory processes (37), particularly activation and adhesion of isolated human neutrophils $(46,50)$. Since we used a reconstituted assay, it is likely that E-selectin engagement is sufficient to enhance rolling on ICAM1. However, it is possible that other factors are deposited from blood or from rolling cells that may be involved in signaling. Our findings are consistent with the observation that E-selectin engagement alone is not sufficient to induce arrest of rolling neutrophils under flow (47). Taken together, these data suggest that engagement of E-selectin ligands on rolling neutrophils promotes partial activation of $\beta_{2}$ integrins. We speculate that this partial LFA-1 activation results in an intermediate integrin conformation similar to that described by Salas et al. (41).

In the autoperfused flow chamber, the number of firmly adherent cells was very low unless a chemokine was immobilized with E-selectin and ICAM-1. However, several in vitro studies have suggested that leukocyte tethering to and rolling on E-selectin can induce $\beta_{2}$ integrin activation and leukocyte firm adhesion $(20,46,47)$. There are several key differences between these studies and the current study that may explain this discrepancy. Simon et al. showed that when isolated human neutrophils were perfused over a monolayer of E-selectin and ICAM-1 expressing Lcells, many neutrophils were able to become firmly adherent (46). The process of neutrophil isolation is known to cause activation $(13,15,27)$. The in vivo environment is very complex and many other factors that are not present in the autoperfused micro flow chamber may influence leukocyte firm adhesion (47).
It has been shown that the isolated I-domain of LFA-1 is able to support rolling on ICAM-1 without inducing firm adhesion (26). In addition, when LFA-1 was stabilized in an extended conformation with a lowaffinity I-domain, rolling on ICAM-1 was increased while firm adhesion was decreased. Furthermore, when LFA-1 was stabilized in an extended conformation with an open, high-affinity I-domain, all interacting cells were firmly adherent and none rolled, and when the integrin was stabilized in a bent conformation, cells were unable to interact with ICAM-1 at all (41). Consistent with our findings, LFA-1 was converted to an extended conformation through the binding of endothelial-presented chemokines (43). However, full I-domain activation occurred only after extended LFA-1 encountered surface-bound ICAM-1 (43). This raises the possibility that some activating stimuli, like ligation of E-selectin ligands, may induce an intermediate conformation of LFA-1 that is able to support rolling, which may result in firm adhesion through additional stimuli. The intermediate affinity conformation of $\beta_{2}$ integrins induced by E-selectin may promote the high-affinity state that preferentially mediates firm adhesion. This would explain why E-selectin is necessary for normal firm leukocyte adhesion in vivo, even though it is not able to directly induce firm adhesion. If $\mathrm{P}$-selectin is present with a chemokine, cells can be activated and adhere. However, our study provides evidence for a mechanism to enhance the inflammatory response by mediating neutrophil integrin activation via E-selectin independently of chemokines. E-selectin may therefore act in cooperation with other adhesion molecules to arrest rolling leukocytes by partially activating integrins and preparing the rolling neutrophils to become fully activated and firmly adhere to the endothelium.

\section{REFERENCES}

1. Alon R, Kassner PD, Carr MW, Finger EB, Hemler ME, Springer TA. (1995). The integrin VLA-4 supports tethering and rolling in flow on VCAM-1. $J$ Cell Biol 128:1243-1253.

2. Beglova N, Blacklow SC, Takagi J, Springer TA. (2002). Cysteine-rich module structure reveals a fulcrum for integrin rearrangement upon activation. Nat Struct Biol 9:282-287.

3. Berlin C, Bargatze RF, Campbell JJ, von Andrian UH, Szabo MC, Hasslen SR, Nelson RD, Berg EL, Erlandsen SL, Butcher EC. (1995). $\alpha_{4}$ integrins mediate lymphocyte attachment and rolling under physiologic flow. Cell 80:413-422. 
4. Butcher EC. (1991). Leukocyte-endothelial cell recognition: three (or more) steps to specificity and diversity. Cell 67:1033-1036.

5. Campbell JJ, Hedrick J, Zlotnik A, Siani MA. Thompson DA, Butcher EC. (1998). Chemokines and the arrest of lymphocytes rolling under flow conditions. Science 279:381-384.

6. Constantin G, Majeed M, Giagulli C. Piccio L, Kim JY, Butcher EC, Laudanna C. (2000). Chemokines trigger immediate beta 2 integrin affinity and mobility changes: differential regulation and roles in lymphocyte arrest under flow. Immunity 13:759-769.

7. Damiano ER, Westheider J, Tozeren A, Ley K. (1996). Variation in the velocity, deformation, and adhesion energy density of leukocytes rolling within venules. Circ Res 79:1122-1130.

8. DiVietro JA, Smith MJ, Smith BR, Petruzzelli L. Larson RS, Lawrence MB. (2001). Immobilized IL8 triggers progressive activation of neutrophils rolling in vitro on $\mathrm{P}$-selectin and intercellular adhesion molecule-1. J Immunol 167:4017-4025.

9. Dunne JL, Ballantyne CM, Beaudet AL, Ley K. (2002). Control of leukocyte rolling velocity in TNFalpha-induced inflammation by LFA-1 and Mac-1. Blood 99:336-341.

10. Dustin, ML. (2001). Beta-2 integrins and their ligands in inflammation. In: Physiology of Inflammation (K. Ley, Ed.). Oxford University Press. New York, 242262.

11. Eppihimer MJ, Wolitzky B, Anderson DC, Labow MA. Granger DN. (1996). Heterogeneity of expression of E- and P-selectins in vivo. Circ Res 79:560-569.

12. Faust N, Varas F, Kelly LM, Heck S, Graf T. (2000). Insertion of enhanced green fluorescent protein into the lysozyme gene creates mice with green fluorescent granulocytes and macrophages. Blood 96:719-726.

13. Forsyth KD, Levinsky RJ. (1990). Preparative procedures of cooling and re-warming increase leukocyte integrin expression and function on neutrophils. J Immunol Methods 128:159-163.

14. Gerszten RE, Garcia-Zepeda EA, Lim YC, Yoshida M, Ding HA, Gimbrone MA Jr, Luster AD, Luscinskas FW, Rosenzweig A. (1999). MCP-1 and IL-8 trigger firm adhesion of monocytes to vascular endothelium under flow conditions. Nature 398:718-723.

15. Glasser L, Fiederlein RL. (1990). The effect of various cell separation procedures on assays of neutrophil function: a critical appraisal. Am J Clin Pathol 93:662-669.

16. Gopalan PK, Smith CW, Lu H, Berg EL, McIntire LV, Simon SI. (1997). Neutrophil CD18-dependent arrest on intercellular adhesion molecule 1 (ICAM-1) in shear flow can be activated through L-selectin. $J$ Immunol 158:367-375.

17. Grabovsky V, Feigelson S, Chen C, Bleijs DA, Peled A, Cinamon G, Baleux F, renzana-Seisdedos F, Lapidot T, van KY, Lobb RR, Alon R. (2000). Subsecond induction of $\alpha_{4}$ integrin clustering by immobilized chemokines stimulates leukocyte tethering and rolling on endothelial vascular cell adhesion molecule 1 under flow conditions. J Exp Med 192:495-506.

18. Hafezi-Moghadam A, Thomas KL, Cornelssen C. (2004). A novel mouse-driven ex vivo flow chamber for the study of leukocyte and platelet function. Am J Physiol Cell Physiol 286:C876-C892.

19. Hattori R. Hamilton KK. Fugate RD, McEver RP. Sims PJ. (1989). Stimulated secretion of endothelial von Willebrand factor is accompanied by rapid redistribution to the cell surface of the intracellular granule membrane protein GMP-140. J Biol Chem 264:77687771 .

20. Hentzen E, McDonough D, McIntire L, Smith CW, Goldsmith HL, Simon SI. (2002). Hydrodynamic shear and tethering through E-selectin signals phosphorylation of p38 MAP kinase and adhesion of human neutrophils. Ann Biomed Eng 30:987-1001.

21. Jung U, Ley K. (1997). Regulation of E-selectin, Pselectin, and intercellular adhesion molecule 1 expression in mouse cremaster muscle vasculature. Microcirculation 4:311-319.

22. Jung U, Ley K. (1999). Mice lacking two or all three selectins demonstrate overlapping and distinct functions for each selectin. J Immunol 162:6755-6762.

23. Jung U, Norman KE, Scharffetter-Kochanek K, Beaudet AL, Ley K. (1998). Transit time of leukocytes rolling through venules controls cytokine-induced inflammatory cell recruitment in vivo. J Clin Invest 102:1526-1533.

24. Jung U, Norman KE, Scharffetter-Kochanek K, Beaudet AL, Ley K. (1998). Transit time of leukocytes rolling through venules controls cytokine-induced inflammatory cell recruitment in vivo. J Clin Invest 102:1526-1533.

25. Kim MB, Sarelius IH. (2004). Role of shear forces and adhesion molecule distribution on P-selectinmediated leukocyte rolling in postcapillary venules. Am J Physiol Heart Circ Physiol 287:H2705-H2711.

26. Knorr R, Dustin ML. (1997). The lymphocyte function-associated antigen $1 \mathrm{I}$ domain is a transient binding module for intercellular adhesion molecule (ICAM)-1 and ICAM-3 in hydrodynamic flow. J Exp Med 186:719-730.

27. Kuijpers TW, Tool AT, van der Schoot CE, Ginsel LA, Onderwater JJ, Roos D, Verhoeven AJ. (1991). Membrane surface antigen expression on neutrophils: a reappraisal of the use of surface markers for neutrophil activation. Blood 78:1105-1111.

28. Kunkel EJ, Ley K. (1996). Distinct phenotype of E-selectin-deficient mice. E-selectin is required for slow leukocyte rolling in vivo. Circ Res 79:11961204.

29. Lawrence MB, McIntire LV, Eskin SG. (1987). Effect of flow on polymorphonuclear leukocyte/endothelial cell adhesion. Blood 70:1284-1290.

30. Lawrence MB, Smith CW, Eskin SG, McIntire LV. (1990). Effect of venous shear stress on 
CD18-mediated neutrophil adhesion to cultured endothelium. Blood 75:227-237.

31. Lawrence MB, Springer TA. (1991). Leukocytes roll on a selectin at physiologic flow rates: distinction from and prerequisite for adhesion through integrins. Cell 65:859-873.

32. Ley K. (2003). Arrest chemokines. Microcirculation. 10:289-295.

33. Ley K, Allietta M, Bullard DC, Morgan S. (1998). Importance of E-selectin for firm leukocyte adhesion in vivo. Circ Res 83:287-294.

34. Long DS, Smith ML, Pries AR, Ley K, Damiano ER. (2004). Microviscometry reveals reduced blood viscosity and altered shear rate and shear stress profiles in microvessels after hemodilution. Proc Natl Acad Sci US A 101:10060-10065.

35. Milstone DS, Fukumura D, Padgett RC, O'Donnell PE, Davis VM, Benavidez OJ, Monsky WL, Melder RJ, Jain RK, Gimbrone MA Jr. (1998). Mice lacking E-selectin show normal numbers of rolling leukocytes but reduced leukocyte stable arrest on cytokine-activated microvascular endothelium. Microcirculation 5:153171.

36. Norman KE. (2001). An effective and economical solution for digitizing and analyzing video recordings of the microcirculation. Microcirculation 8:243-249.

37. Ono K, Han J. (2000). The p38 signal transduction pathway: activation and function. Cell Signal 12:113.

38. Ramos CL, Kunkel EJ, Lawrence MB, Jung U, Vestweber D, Bosse R, McIntyre KW, Gillooly KM, Norton CR, Wolitzky BA, Ley K. (1997). Differential effect of E-selectin antibodies on neutrophil rolling and recruitment to inflammatory sites. Blood 89:3009-3018.

39. Reinhardt PH, Kubes P. (1998). Differential leukocyte recruitment from whole blood via endothelial adhesion molecules under shear conditions. Blood 92:46914699 .

40. Salas A, Shimaoka M, Chen S, Carman CV, Springer T. (2002). Transition from rolling to firm adhesion is regulated by the conformation of the I domain of the integrin lymphocyte function-associated antigen1. J Biol Chem 277:50255-50262.

41. Salas A, Shimaoka M, Kogan AN, Harwood C, von Andrian UH, Springer TA. (2004). Rolling adhesion through an extended conformation of integrin $\alpha_{L} \beta_{2}$ and relation to $\alpha \mathrm{I}$ and $\beta$ I-like domain interaction. Immunity 20:393-406.
42. Scharffetter-Kochanek K, Lu H, Norman K, van NN, Munoz F, Grabbe S, McArthur M, Lorenzo I, Kaplan S, Ley K, Smith CW, Montgomery CA, Rich S, Beaudet AL. (1998). Spontaneous skin ulceration and defective $\mathrm{T}$ cell function in CD18 null mice. $J$ Exp Med 188:119-131.

43. Shamri R, Grabovsky V, Gauguet JM, Feigelson S, Manevich E, Kolanus W, Robinson MK, Staunton DE, von Andrian UH, Alon R. (2005). Lymphocyte arrest requires instantaneous induction of an extended LFA-1 conformation mediated by endothelium-bound chemokines. Nat Immunol 6: 497-506.

44. Shimaoka M, Xiao T, Liu JH, Yang Y, Dong Y, Jun CD, McCormack A, Zhang R, Joachimiak A, Takagi J, Wang JH, Springer TA. (2003). Structures of the $\alpha_{L}$ I domain and its complex with ICAM-1 reveal a shape-shifting pathway for integrin regulation. Cell 112:99-111.

45. Simon SI, Green CE. (2004). Molecular mechanics and dynamics of leukocyte recruitment during inflammation. Annu Rev Biomed Eng 7:3.1-3.35.

46. Simon SI, Hu Y, Vestweber D, Smith CW. (2000) Neutrophil tethering on E-selectin activates $\beta_{2}$ integrin binding to ICAM-1 through a mitogen-activated protein kinase signal transduction pathway. J Immunol 164:4348-4358.

47. Smith ML, Olson TS, Ley K. (2004). CXCR2- and Eselectin-induced neutrophil arrest during inflammation in vivo. J Exp Med 200:935-939.

48. Smith ML, Smith MJ, Lawrence MB, Ley K. (2002). Viscosity-independent velocity of neutrophils rolling on $\mathrm{P}$-selectin in vitro or in vivo. Microcirculation 9:523-536.

49. Smith ML, Sperandio M, Galkina EV, Ley K. (2004). Autoperfused mouse flow chamber reveals synergistic neutrophil accumulation through P-selectin and E-selectin. J Leukoc Biol 76:985993.

50. Smolen JE, Petersen TK, Koch C, O'Keefe SJ, Hanlon WA, Seo S, Pearson D, Fossett MC, Simon SI. (2000). L-selectin signaling of neutrophil adhesion and degranulation involves p38 mitogenactivated protein kinase. J Biol Chem 275:1587615884

51. Tong L, Pav S, White DM, Rogers S, Crane KM, Cywin CL, Brown ML, Pargellis CA. (1997). A highly specific inhibitor of human p38 MAP kinase binds in the ATP pocket. Nat Struct Biol 4:311-316. 